\title{
Computed tomography angiography in blunt cerebrovascular injury: navigating through perspectives and diagnostics
}

\author{
Andrea Matteucci ${ }^{1,2} \cdot$ Giuseppe Sangiorgi $^{3} \cdot$ Carolina Giordano $^{4} \cdot$ Giuseppe Biondi-Zoccai $^{5,6}$ (1)
}

Received: 28 October 2021 / Revised: 28 October 2021 / Accepted: 18 November 2021 / Published online: 29 January 2022

(c) The Author(s), under exclusive licence to European Society of Radiology 2022

You're gonna need a bigger boat

Roy Scheider, Jaws (1975)

\section{Blunt cerebrovascular injury}

Blunt cerebrovascular injury (BCVI) remains a key cause of mortality and morbidity, thus proving a contemporary discussion topic and a source of study due to the paucity of available recommendations [1]. It consists of non-penetrating damage to the vertebral or carotid arteries due to stretching of the vessel following sudden and abrupt movements of flexion, extension, or rotation of the head, either traumatic or not. The consequences are intimal tear with exposure of subintimal layers to the blood flow, consequent formation of thrombi with possible lumen occlusion, or haematoma of the vessel wall. A further possible evolution of the lesion is the formation of a pseudoaneurysm. Both thrombus formation and lumen occlusion are responsible for ischaemia and

This comment refers to the article available at https://doi.org/10. 1007/s00330-021-08379-7.

Giuseppe Biondi-Zoccai

giuseppe.biondizoccai@uniroma1.it

1 Department of Experimental Medicine, Tor Vergata University of Rome, Rome, Italy

2 Division of Cardiology, San Filippo Neri Hospital, Via Martinotti, 20, 00135 Rome, Italy

3 Department of Biomedicine and Prevention, Tor Vergata University of Rome, Rome, Italy

4 Neuroradiology Unit, Department of Diagnostic Imaging, Oncological Radiotherapy, and Hematology, Fondazione Policlinico Universitario Agostino Gemelli IRCCS and Università Cattolica del Sacro Cuore, Rome, Italy

5 Department of Medical-Surgical Sciences and Biotechnologies, Sapienza University of Rome, Corso della Repubblica 79, 04100 Latina, Italy

6 Mediterranea Cardiocentro, Napoli, Italy stroke, whose diagnosis may be delayed by presentation at onset, and sometimes by the availability and reliability of radiological examinations.

\section{Digital subtraction angiography vs computed tomography angiography}

To date, the two main used diagnostic approaches are digital subtraction angiography (DSA), the gold standard in the detection of BCVI, and computed tomography angiography (CTA). In order to help clinicians in choosing the most appropriate test, several screening tools have been developed to identify patients at risk of BCVI and limit the use of angiography (Denver criteria and modified Memphis criteria are the most commonly used ones) [2,3]. Comparing DSA to CTA, there are higher risks of procedure-related complications, such as stroke, pseudoaneurysm, and haematoma. Moreover, DSA is not always available, and a delay in treatment may depend on its choice [4].

Therefore, CTA gained prominence in BCVI diagnosis; widespread availability in the emergency setting makes it ideal as a primary imaging tool. In a meta-analysis by Roberts et al. [5], the pooled sensitivity and specificity for BCVI detection with CTA versus DSA were $66 \%$ and $97 \%$, respectively. The authors hypothesised that the lower sensitivity of CTA might be due to an implicit variation in diagnostic threshold across trauma centres. The lack of a standardised diagnostic pathway limited the use of CTA due to the high number of false negative results for BCVI. But CTA was superior to DSA when patients were selected according to the Denver screening criteria. Malhotra et al. showed CTA is the optimal imaging strategy in high-risk BCVI patients [6]. Specifically, and despite the low sensitivity of CTA, their cost-effectiveness analysis demonstrates the superiority of this strategy over DSA in the setting of high-risk patients.

All studies considered were conducted on 16/32-channel CTA, which may have represented a further disadvantage for adequate sensitivity of the methodology. A subsequent 
Fig. 1 Use of digital subtraction angiography and computed tomography (CT) angiography in blunt cerebrovascular injury (BCVI). GCS, Glasgow Coma Scale; MRI, magnetic resonance imaging; MS, mental state; TBI, traumatic brain injury; TIA, transient ischemic attack

\section{Blunt Cerebrovascular Injury - Diagnostic Pathway}

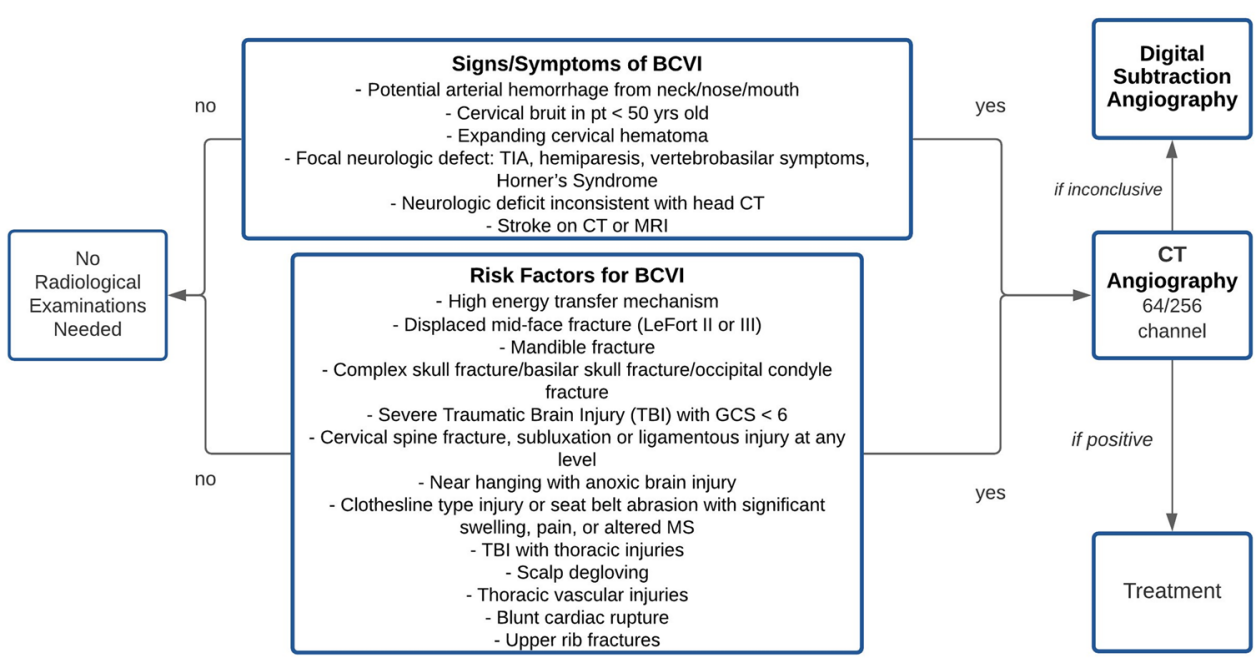

study showed how 64-channel CTA significantly improved sensitivity by $68 \%$ compared to the $51 \%$ previously reported for 32-channel CTA [7]. Nevertheless, the majority of false positives $(62 \%)$ still occurred in patients at low risk of events. It is safe to assume that improved CT equipment technology will lead to improved diagnostic performance in, the true Achilles' heel of CTA: low-risk patients. But while the results of Paulus et al. suggest this idea, the results of a recent meta-analysis undermine this premise.

Kik et al. started from the above results and hypothesised that 64-channel CTA could have comparable diagnostic accuracy to DSA and could therefore replace it. The diagnostic accuracy estimates for CTA compared to DSA for any form of BCVI were $64 \%$ for sensitivity and $95 \%$ for specificity. Estimates for individual districts were also reported, showing an even higher sensitivity of CTA in BCVI $\mathrm{I}_{\text {carotid }}$ and $\mathrm{BCVI}_{\text {vertebral }}\left(70 \%\right.$ sensitivity, $98 \%$ specificity in $\mathrm{BCVI}_{\text {carotid}}$, and $70 \%$ sensitivity, $99 \%$ specificity in $\mathrm{BCVI}_{\text {vertebral }}$ ). In contrast to previous studies, however, subgroup analysis and meta-regression show that there was no significant difference between studies based on publication year and the number of CT detector rows when calculating combined pooled sensitivity and specificity of CTA for the detection of any BCVI. The discrepancy was found only in the analysis by artery and by patient, where the sensitivity of $\leq 16$-channel CTA was significantly lower than $\geq 16$-channel CTA $(63.1 \%$ vs $70.3 \%$ ). A valid interpretation of the results is provided by the authors, who highlight a possible selection bias. Only studies in which patients underwent both approaches were analysed, which may have led to an underrepresentation of patients with high-grade BCVI or even those with lowgrade dissections who are only screened when severe and/ or delayed neurological symptoms occur.

\section{"We need a bigger boat"}

We must therefore confront the evidence: CTA with more channels (32-64 channels) did not increase diagnostic accuracy compared to CTA with fewer channels $(\leq 16$ channels). However, this is true when comparing these two CTA-specific setups, which to date are outdated and obsolete. This is why the recent results reported by Kik et al. can only be our starting point, and their interpretation as suggested by the authors is not unambiguous [8]. In the digital era, implementation of machine learning, and continuous improvements in technological innovation, represented by newer CT scanners with 256-detector rows, low $\mathrm{kVp}$ imaging, multi-energy reconstruction and different post-processing 3D reconstruction, are the new standards. Thus, it is pivotal to conduct research with last-generation CT scanners, using advanced reconstruction software where available. Extending the use of multi-detector CTA to all centres, adopting protocols which assess this screening methodology routinely to all suspected cases, should shortly bridge the sensitivity gap between CTA and DSA (Fig. 1) [9]. Indeed, it is pivotal to also consider CTA and DSA as synergistic, in a logic of hybrid imaging suitable for more comprehensive and multidimensional diagnostic and prognostic assessment [10].

Funding The author states that this work has not received any funding.

\section{Declarations}

Guarantor The scientific guarantor of this publication is Giuseppe Biondi-Zoccai. 
Conflict of interest The authors of this manuscript declare relationships with the following companies: Giuseppe Biondi-Zoccai has consulted for Cardionovum, Innovheart, Meditrial, Opsens Medical, and Replycare.

Statistics and biometry No complex statistical methods were necessary for this paper.

Informed consent Written informed consent was not required for this study because it is an editorial.

Ethical approval Institutional Review Board approval was not required because this is an editorial.

\section{Methodology}

- Editorial comment

\section{References}

1. Brommeland T, Helseth E, Aarhus M et al (2018) Best practice guidelines for blunt cerebrovascular injury (BCVI). Scand J Trauma Resusc Emerg Med 26:90

2. Bensch FV, Varjonen EA, Pyhältö TT, Koskinen SK (2019) Augmenting Denver criteria yields increased BCVI detection, with screening showing markedly increased risk for subsequent ischemic stroke. Emerg Radiol 26:365-372

3. Ciapetti M, Circelli A, Zagli G et al (2010) Diagnosis of carotid arterial injury in major trauma using a modification of Memphis criteria. Scand J Trauma Resusc Emerg Med 18:61

4. Willinsky RA, Taylor SM, TerBrugge K, Farb RI, Tomlinson G, Montanera W (2003) Neurologic complications of cerebral angiography: prospective analysis of 2,899 procedures and review of the literature. Radiology 227:522-528

5. Roberts DJ, Chaubey VP, Zygun DA et al (2013) Diagnostic accuracy of computed tomographic angiography for blunt cerebrovascular injury detection in trauma patients: a systematic review and meta-analysis. Ann Surg 257:621-632

6. Malhotra A, Wu X, Kalra VB, Schindler J, Matouk CC, Forman HP (2016) Evaluation for blunt cerebrovascular injury: review of the literature and a cost-effectiveness analysis. AJNR Am J Neuroradiol 37:330-335

7. Paulus EM, Fabian TC, Savage SA et al (2014) Blunt cerebrovascular injury screening with 64-channel multidetector computed tomography: more slices finally cut it. J Trauma Acute Care Surg $76: 279-283$

8. Kik CC, Sloof WM, Moayeri N, de Jong PA, Muijs SPJ, Öner FC (2021) Diagnostic accuracy of computed tomography angiography (CTA) for diagnosing blunt cerebrovascular injury in trauma patients: a systematic review and meta-analysis. Eur Radiol. https://doi.org/10.1007/s00330-021-08379-7

9. Alfanek Z, Herzog A, Taylor $\mathrm{N}$ et al (2021) Evaluating the routine use of head computed tomography angiography in blunt cerebrovascular trauma. J Surg Res 269:129-133

10. Bajaj R, Garcia-Garcia HM, Courtney BK et al (2021) Multimodality intravascular imaging for guiding coronary intervention and assessing coronary atheroma: the Novasight Hybrid IVUSOCT system. Minerva Cardiol Angiol. https://doi.org/10.23736/ S2724-5683.21.05532-0

Publisher's note Springer Nature remains neutral with regard to jurisdictional claims in published maps and institutional affiliations. 